\title{
Influencia de la motivación hacia el deporte en el consumo de alcohol, tabaco y cannabis de adolescentes escolares
}

\author{
Influence of motivation towards sports in alcohol, tobacco \\ and cannabis consumption in adolescent students
}

\author{
Pablo Usán ${ }^{1}$ \\ Carlos Salavera ${ }^{2}$ \\ Universidad de Zaragoza, España
}

\begin{abstract}
Resumen. Uno de los problemas más graves a los que se enfrenta la sociedad actual resulta el consumo de drogas por parte de la población infantil y juvenil. La práctica deportiva puede actuar como factor de prevención del consumo; se la considera como uno de los hábitos más representativos de un estilo de vida saludable. Por ello, el objetivo del estudio es analizar la relación e influencia de la motivación hacia la práctica deportiva en el consumo de alcohol, tabaco y cannabis de adolescentes deportistas escolares. Los resultados desprenden influencias significativas de motivaciones intrínsecas, extrínsecas y falta de motivación en las diferentes prevalencias de consumo en unos patrones de comportamiento más o menos autodeterminados y adaptativos.
\end{abstract}

Palabras clave. Motivación, consumo, drogas, adolescentes, deporte.

Abstract. One of the most serious problems faced by our society is the use of drugs by young people. Sports practice can act as a preventive factor being one of the most representative habits of a healthy lifestyle. Therefore, the objective of the study is to analyze the relationship and influence of motivation towards sports practice in the consumption of alcohol, tobacco and cannabis of school sports adolescents. The results reveal significant influences of intrinsic, extrinsic motivations and lack of motivation in the different prevalence of consumption in more or less self-determined and adaptive behavior patterns.

Keywords. Motivation, drugs, consumption, adolescent, sport.

${ }^{1}$ Pablo Usán. Facultad de Educación, Universidad de Zaragoza, España. Dirección portal: C/ Pedro Cerbuna,12.50009 Zaragoza, España. E-mail: pablousan@hotmail.com

${ }^{2}$ Carlos Salavera. Facultad de Educación, Universidad de Zaragoza, España.E-mail: salavera@unizar.es 


\section{Introducción}

En la actualidad, el consumo de drogas, tanto las de tipo legal (alcohol, tabaco, tranquilizantes, etc.) como las ilegales (marihuana, hachís, cocaína, etc.) obtienen unos altos porcentajes de consumo entre la población. Además, existe elevada incidencia en numerosos problemas para la salud de las personas que las utilizan (infartos, cáncer, afecciones...), así como una creciente mortalidad en muchos de sus consumidores habituales (Becoña, 2007).

Dicha problemática concierne a una preocupación social y ha conducido a una profunda transformación y adecuación de las estructuras y recursos provenientes desde la administración. Lo anterior con la finalidad de hacer frente a las necesidades planteadas desde diferentes ámbitos como la prevención de consumo, el control de la oferta de sustancias adictivas y, de la misma manera, la asistencia sanitaria y social.

De este modo, el uso de drogas en España ha experimentado un aumento significativo desde principios de la década de los noventa hasta la actualidad, ligado un considerable incremento de las consecuencias sociales, sanitarias y culturales (Baquero, Pastor, \& Llopis, 2003; Calafat, Juan, Becoña, Fernández, \& Gil, 2002; Oliva, Parra, \& Sánchez-Queija, 2006). Así, las drogas constituyen uno de los problemas más importantes con los que se enfrenta la sociedad; resulta especialmente alarmante cuando el consumo de drogas se produce en población adolescente, no solo por los problemas inmediatos que pueden generarle sino, también, por la consolidación de patrones de consumo y hábitos desadaptativos que conlleva su inicio desde edades prontas.

Desde el Plan Nacional Sobre Drogas (PNSD, 2016), se alude a un aumento significativo del consumo de drogas entre los adolescentes, así como variaciones en la edad de inicio (situada en los 13.2 años). Las sustancias más consumidas por los adolescentes españoles resultan ser, por orden descendente, alcohol, tabaco y cannabis. A la edad media de los 16 años, un $67.8 \%$ de los adolescentes se consideran consumidores ocasionales de alcohol; un 39.4\%, de tabaco y un $8.9 \%$ afirma ser consumidor habitual de cannabis.
Otro aspecto a destacar es el policonsumo entre los adolescentes; es decir, el hecho de que es frecuente que incluyan más de un tipo de sustancia en sus patrones de consumo. Por ejemplo, los consumidores habituales de cocaína recurren, además de tabaco y alcohol, a otras drogas ilegales como cannabis o alucinógenos (Calafat et al., 2002). De este modo, existe una situación compleja en la que un gran porcentaje de la población consume no solo de una sustancia, sino de varias al mismo tiempo, lo que podría acarrear consecuencias indeseables. Numerosos estudios insisten en que se trata de un problema complejo en el que interaccionan variables de muy diversa índole (Bravo, Echeburúa, \& Aizpiri, 2008; Espada, Méndez, Griffin, \& Botvin, 2003; Gilman et al., 2009).

Sin duda, resulta una situación preocupante si se tiene en cuenta la adolescencia como periodo vital y paso previo a la edad adulta. En este, tienen lugar los primeros contactos con las sustancias adictivas que pueden derivar en la consolidación de patrones de consumo perdurables en el tiempo que podrían acarrear problemas que afecten de manera determinante en la edad adulta (Alcalá, Azañas, Moreno, \& Gálvez, 2002; Gómez-Fraguela, Fernández, Romero, \& Luengo, 2008).

Por otro lado, la práctica de la actividad físicadeportiva está considerada como uno hábito representativo dentro de una línea de estilo saludable con innumerables ventajas para las personas que la practican (Cruz, Fernández, \& González, 2007). Desde esta perspectiva, numerosas investigaciones señalan que aquellos adolescentes deportistas que realizan una práctica habitual de actividad físico-deportiva se inician más tarde en el consumo de drogas (De Vries et al., 2006; Rodríguez, De Abajo, \& Márquez, 2004; García, Carrillo, Fernández, \& Sánchez, 2006; Nistal, Prieto, Del Valle, \& González, 2003; Pastor, Balaguer, \& García-Merita, 2006; Warren et al., 2008).

Un aspecto esencial de cualquier práctica físicodeportiva es la motivación de sus participantes; esta puede determinar su adherencia y evitación del abandono deportivo (García-Calvo, 2006; Macarro, Romero, \& Torres, 2008; Mañas, Salvador, Boada, \& Agulló, 2007). 
Así, la motivación hacia la práctica deportiva es una de las variables psicológicas más estudiadas en deportistas.

Desdelaperspectivadelateoríadelaautodeterminación (TAD) (Deci \& Ryan, 2002), resalta un continuo que abarca diferentes grados de autodeterminación en la conducta, desde la no autodeterminada hasta la más autodeterminada (Moreno \& Martínez, 2006). En este recorrido, se abarcan tres tipos fundamentales de motivación, intrínseca, extrínseca y amotivación, reguladas por el sujeto de manera interna o externa. La motivación intrínseca, hace referencia a motivos propios del sujeto en el desarrollo de la actividad suponiendo el compromiso, diversión y disfrute siendo un fin en sí misma (Deci \& Ryan, 2000). La motivación extrínseca alude a motivos de práctica ajenos a la propia actividad o deporte siendo una tarea externa a la actividad que se está realizando. La amotivación hace referencia a la falta de motivación para desarrollar una tarea o actividad en un contexto determinado. Por lo tanto, la motivación es un elemento clave para lograr la adherencia y el compromiso en el deporte (Iso-Ahola \& St.Clair, 2008) representando una disposición psicológica de deseo y decisión de seguir participando en él (Scanlan, Russell, Beals, \& Scanlan, 2003).

Numerosos estudios relacionan la motivación con el consumo de sustancias. Moreno, GonzálezCutre y Cervelló (2008) determinan la relación entre motivaciones intrínsecas propias a la actividad deportiva y ausencia de consumos prediciendo hábitos saludables en adolescentes deportistas. Otros trabajos asocian un menor consumo de tabaco y alcohol con una alta motivación intrínseca, menor amotivación, mayor disfrute y creencias de éxito de la actividad físico-deportiva (Inglés, Delgado, Bautista, Torregrosa, Espada, García-Fernández et al., 2007; Llamas, 2009; Usán, Salavera, Murillo \& Álvarez, 2017). En una misma línea, Leyton, Jiménez, Domínguez y Corzo (2012) manifiestan la relación de una ausencia de consumo de tabaco en relación con motivaciones intrínsecas y autorreferenciales unidas a hábitos de salud favorables como un adecuado descanso y alimentación.

En una línea opuesta, los trabajos de Moreno et al. (2008) determinan que aquellos deportistas que adquieren una motivación menos autodeterminada predicen significativamente hábitos de consumo de sustancias durante la adolescencia. Moreno y Cervelló (2009) muestran relaciones positivas entre motivaciones puramente extrínsecas y falta de motivación hacia la práctica deportiva con consumos de alcohol en una línea de comportamientos más desadaptativos.

Villalbí, Suelves, Saltó y Cabezas (2011) manifiestan la implicación y consecuencias del consumo de cannabis en el rendimiento deportivo de los futbolistas (mayor fatigabilidad, menor recuperación física, dificultades cardíacas...); estas merman su motivación intrínseca hacia la práctica deportiva en una línea de comportamientos menos autodeterminados.

Pese a todo ello, y siguiendo a Moreno et al. (2008), resultan necesarios más estudios que indaguen las relaciones entre la práctica de la actividad físicodeportiva y el consumo de drogas.

De este modo, el principal objetivo de este estudio es analizar la relación e influencia de la motivación deportiva en el consumo de alcohol, tabaco y cannabis en deportistas adolescentes escolares con las siguientes hipótesis:

a) Existencia de relaciones significativas entre mayores motivaciones intrínsecas y menores consumos de alcohol, tabaco y cannabis en una línea más de conductas más autodeterminada.

b) Existencia de relaciones significativas entre mayores motivaciones extrínsecas y falta de motivación con mayores consumos de alcohol, tabaco y cannabis en una línea de conductas menos autodeterminada.

\section{Método}

\section{Diseño}

Siguiendo a Ato, López y Benavente (2013), el estudio es de tipo ex post-facto, de carácter prospectivo, con un diseño descriptivo simple, respondiendo a un muestreo aleatorio simple.

Muestra

El grupo estuvo compuesto por 626 adolescentes deportistas masculinos, pertenecientes a 14 clubes de 
Tabla 1

Edad de los adolescentes participantes

\begin{tabular}{ccc}
\hline Edad & $N$ & $\%$ \\
\hline 14 años & 102 & 16.2 \\
15 años & 144 & 23.0 \\
16 años & 138 & 22.0 \\
17 años & 98 & 15.6 \\
18 años & 86 & 13.7 \\
19 años & 58 & 9.2 \\
\hline
\end{tabular}

fútbol, con edades comprendidas entre los 14 y 19 años, todos ellos federados y activos en la práctica deportiva (ver tabla 1).

\section{Instrumentos de evaluación}

Para recoger la información y lograr una aproximación al objetivo establecido se utilizaron dos cuestionarios.

Para percibir los motivos de práctica de los adolescentes deportistas se utilizó una versión traducida y adaptada de la Sport Motivation Scale (Pelletier, Fortier, Vallerand, Tuson, Brière, \& Blais, 1995), validada y traducida al español por Núñez, Martín-Albo, Navarro y González (2006). Esta escala se compone de 28 ítems repartidos en tres grandes motivaciones con sus respectivas subvariables de igual número de ítems (4): motivación intrínseca, orientada hacia la práctica (p. ej. por la satisfacción que me produce realizar algo excitante) $(\alpha=.82)$, aprendizaje (p. ej. por la satisfacción de aprender algo más sobre este deporte) $(\alpha=.76)$ y perfeccionamiento (p. ej. por la satisfacción que siento mejorando algunos de mis puntos flacos) $(\alpha=$ .78); motivación extrínseca, identificada (p. ej. porque en mi opinión es una de las mejores formas de conocer gente) $(\alpha=.72)$, introyectada (p. ej. porque debo participar para sentirme bien conmigo mismo) $(\alpha=.78)$ y externa ( $\mathrm{p}$. ej. porque me permite ser valorado por la gente que conozco) $(\alpha=.79)$ y, por último, amotivación (p. ej. no lo sé, siento que no soy capaz de tener éxito en este deporte) $(\alpha=.81)$ o, lo que es lo mismo, la falta de motivación hacia la práctica deportiva. La fiabilidad del instrumento original denota un alfa de Cronbach de .750.
Con la finalidad de percibir el consumo por parte de los futbolistas adolescentes, se utilizó una versión adaptada de la Encuesta Estatal Sobre Uso de Drogas en Enseñanzas Secundarias (ESTUDES, 2016), promovida por la delegación del gobierno para el Plan Nacional sobre Drogas (PNSD). Se tomaron las categorías generales del consumo de alcohol $(\alpha$ $=.77)$ tabaco $(\alpha=.74)$ y cannabis $(\alpha=.75)$ con tres opciones de respuesta: consumo habitual, ocasional o ausencia del mismo, mostrando una consistencia interna de .768 .

\section{Procedimiento}

Para la realización del estudio se contó con la aprobación de los 14 clubes de fútbol y de los padres/ tutores de los futbolistas por medio de consentimiento informado acerca de los fines de la investigación. Un día de la semana, acordado previamente con los entrenadores, se llevó a cabo la cumplimentación de los cuestionarios en cada uno de los clubes. Una vez recogidos los datos, se procesaron y analizaron mediante el programa estadístico IBM SPSS v22.0. Todos los sujetos y sus padres/tutores fueron previamente informados de la naturaleza del estudio, participaron de manera voluntaria, respetando las directrices éticas de la Declaración de Helsinki (AMM, 2000) en todos sus términos.

Análisis de datos

Se procedió a realizar una estadística descriptiva para hallar los datos sociodemográficos de las diferentes 
variadas estudiadas. Posteriormente, se realizaron correlaciones entre las variables motivación deportiva y consumo. Se estableció un análisis de ecuaciones entre las diferentes motivaciones y consumos. Finalmente, mediante el análisis de cluster, se clasificaron a los sujetos del estudio según sus características específicas, en diferentes grupos cuyas puntuaciones fueron significativas entre sí. Para todas las operaciones, se tuvo en cuenta un nivel de significación $p \leq .05$.

\section{Resultados}

\section{Variables descriptivas}

Tal y como se observa en la tabla 2, las diferentes motivaciones intrínsecas (orientadas hacia la práctica, aprendizaje y perfeccionamiento) obtuvieron mayores puntuaciones que las motivaciones extrínsecas, emergiendo la motivación introyectada como variable de mayor media entre ellas. La amotivación, o falta de motivación, resultó la menos puntuada por los deportistas adolescentes.

Por otra parte, atendiendo a las prevalencias de consumo de drogas, el alcohol se denotó como la sustancia más consumida entre los deportistas adolescentes, tanto a nivel habitual $(N=148 ; 23.6$ $\%$ ) como ocasional $(N=344 ; 54.9 \%)$, seguido por el tabaco (véase tabla 3). El consumo de cannabis resultó ser menos acentuado que las dos anteriores, con escasas prevalencias de consumo habitual $(N=44 ; 7.0$ \%) así como ausencia del mismo $(N=445 ; 71.0 \%)$.

Relación entre la motivación deportiva y el consumo de alcohol, tabaco y cannabis

Se analizaron las variables deportivas y de consumo; se apreciaron correlaciones significativas entre varias de ellas (véase tabla 4). Así, destacó la significación de motivaciones intrínsecas, especialmente las orientadas hacia la práctica y el aprendizaje, con el consumo de

Tabla 2

Variables descriptivas de la motivación

\begin{tabular}{lcccc}
\hline Motivación deportiva & Media & $D T$ & Rango & $\alpha$ \\
\hline Intrínseca practicar & 4.04 & .69625 & 4.00 & .82 \\
Intrínseca aprender & 3.81 & .79518 & 4.00 & .76 \\
Intrínseca perfeccionar & 3.90 & .74248 & 3.75 & .78 \\
Extrínseca externa & 2.49 & .89661 & 4.00 & .79 \\
Extrínseca identificada & 3.51 & .83687 & 4.00 & .72 \\
Extrínseca introyectada & 3.73 & .68084 & 4.00 & .78 \\
Amotivación & 1.72 & .76653 & 4.00 & .81 \\
\hline
\end{tabular}

Tabla 3

Frecuencias de consumo de alcohol, tabaco y cannabis

\begin{tabular}{lccccccc}
\hline & \multicolumn{2}{c}{ Nunca } & \multicolumn{2}{c}{ Ocasional } & \multicolumn{2}{c}{ Habitual } \\
\cline { 2 - 8 } Sustancia & $N$ & $\%$ & $N$ & $\%$ & $N$ & $\%$ & $\alpha$ \\
\hline Alcohol & 134 & 21.4 & 344 & 54.9 & 148 & 23.6 & .77 \\
Tabaco & 326 & 52.0 & 219 & 34.9 & 81 & 12.9 & .74 \\
Cannabis & 445 & 71.0 & 137 & 21.8 & 44 & 7.0 & .75 \\
\hline
\end{tabular}


Tabla 4

Relación entre variables deportivas y consumos

\begin{tabular}{lccc}
\hline \multirow{2}{*}{ Motivación deportiva } & \multicolumn{3}{c}{ Consumos } \\
\cline { 2 - 4 } & Alcohol & Tabaco & Cannabis \\
\hline Intrínseca practicar & $-.151^{* *}$ & $-.121^{* *}$ & $-.125^{* *}$ \\
Intrínseca aprender & $-.214^{* *}$ & $-.142^{* *}$ & $-.154^{* *}$ \\
Intrínseca perfeccionar & $-.132^{* *}$ & -.055 & -.082 \\
Extrínseca externa & -.044 & -.034 & -.014 \\
Extrínseca identificada & $-.146^{* *}$ & $-.128^{* *}$ & $-.111^{*}$ \\
Extrínseca introyectada & -.058 & $-.092^{*}$ & $-.102^{*}$ \\
Amotivación & $.143^{* *}$ & $.174^{* *}$ & $.197^{* *}$ \\
\hline
\end{tabular}

** La correlación es significativa al nivel .01 (bilateral).

* La correlación es significante al nivel .05 (bilateral).

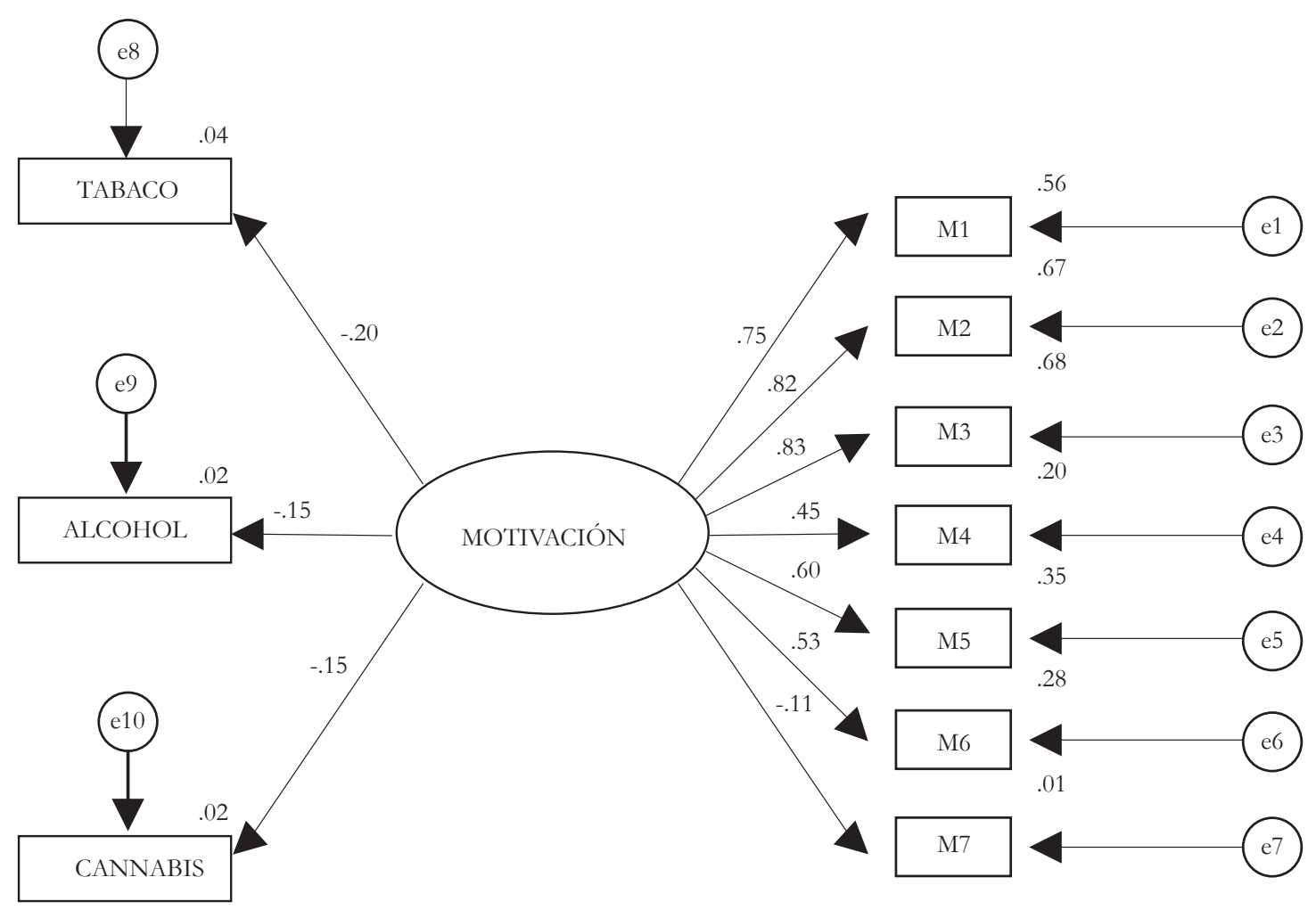

Figura 1. Modelo de ecuaciones estructurales entre la motivación1 y el consumo de alcohol, tabaco y cannabis. M1(Motivación intrínseca hacia la práctica); M2 (Motivación intrínseca hacia el aprendizaje); M3 (Motivación intrínseca hacia el perfeccionamiento); M4 (Motivación extrínseca externa); M5 (Motivación extrínseca identificada); M6 (Motivación extrínseca introyectada); M7 (Amotivación). 
alcohol, tabaco y cannabis, de la misma manera que lo hizo con la motivación extrínseca identificada (-.146**) y la falta de motivación (.143**).

Análisis del modelo entre la motivación y el consumo de alcohol, tabaco y cannabis

En la figura 1 se muestra el resultado del análisis con ecuaciones estructurales mediante el método de extracción de máxima verosimilitud, confirmando la adecuación del modelo compuesto por los constructos planteados en el estudio. En este caso, se observa cómo la motivación presenta relaciones inversas con los consumos (tabaco $r=-.20$; alcohol $r=-.15$; cannabis $r$ $=-.15$ ). Esto indicaría que, a mayor motivación en los adolescentes, menores consumos y viceversa. Por otra parte, todos los factores de motivación presentaron adecuados ajustes con la amotivación, presentando una pequeña relación inversa $(r=-.11)$. Por lo que se refiere al ajuste del modelo, los diversos índices de ajuste resultaron adecuados; de este modo, se puede afirmar que el modelo propuesto acerca de la estructura factorial entre los factores es sustentable: $\chi^{2}(35)=85.678, p<$ $.001 ; \chi^{2} / \mathrm{gl}=2.447 ; \mathrm{CFI}=0.96 ; \mathrm{NFI}=0.94 ; \mathrm{TLI}=$ 0.94; RMSEA $=0.063$, IC 95\% (0.048 - 0.079).

Tabla 5

Distribución de los sujetos en grupos significativos

\begin{tabular}{ccc}
\hline & $N$ & $\%$ \\
\hline GRUPO 1 & 167 & $26.6 \%$ \\
GRUPO 2 & 192 & $30.6 \%$ \\
GRUPO 3 & 267 & $42.6 \%$ \\
\hline
\end{tabular}

Tabla 6

Medias significativas de cada grupo de variables deportivas y consumo

\begin{tabular}{lcccc}
\hline & G.1 & G.2 & G.3 & Media \\
\hline Motivación deportiva & & & & \\
Intrínseca practicar & 3.39 & 4.35 & 4.14 & 4.04 \\
Intrínseca aprender & 3.04 & 4.25 & 3.92 & 3.81 \\
Intrínseca perfeccionar & 3.27 & 4.31 & 4.00 & 3.90 \\
Extrínseca externa & 2.12 & 3.29 & 2.18 & 2.49 \\
Extrínseca identificada & 2.96 & 4.00 & 3.51 & 3.51 \\
Extrínseca introyectada & 3.32 & 4.11 & 3.73 & 3.73 \\
Amotivación & 2.06 & 2.03 & 1.38 & 1.72 \\
Sustancias consumidas & & & & \\
Alcohol & 1.53 & 1.12 & .79 & 1.08 \\
Tabaco & .100 & .52 & .21 & .50 \\
Cannabis & .77 & .36 & .11 & .35 \\
\hline
\end{tabular}


Patrones de conducta entre variables deportivas y consumo de alcohol, tabaco y cannabis

Por último, se clasificó a los participantes en tres grupos homogéneos en función de sus niveles de motivación y consumo realizando un análisis de cluster, el cual tiene por objetivo la clasificación de los individuos en grupos significativos. Tal y como se puede observar en la tabla 5 , se establecieron tres grupos significativos en función de sus medias.

A continuación, se muestran, de manera específica, las diferentes medias de cada variable en función a los tres grupos significativos anteriores (tabla 6).

Primer grupo $(\mathrm{N}=167 ; 26.6 \%)$. Caracterizado por conductas menos adaptativas:Las motivaciones intrínsecas se dispusieron por debajo de la media en detrimento de un mayor consumo de alcohol, tabaco y cannabis, así como prevalencias superiores de amotivación.

Segundo grupo $(N=192 ; 30.6 \%)$. Caracterizado por conductas mixtas: Las variables deportivas de motivación se dispusieron en su mayoría por encima de la media, resultando prevalencias ligeramente superiores a ella en los consumos de alcohol, tabaco y cannabis.

Tercer grupo $(N=267 ; 42.6 \%$ ). Caracterizado por conductas más adaptativas: Las medias de este grupo en motivaciones intrínsecas fueron superiores a la media total resultando las extrínsecas y amotivación por debajo de ella. Todo ello unido a un considerable descenso de las prevalencias de alcohol, tabaco y cannabis por parte de los futbolistas adolescentes que componían este grupo.

\section{Discusión}

El objetivo de la investigación era analizar la relación e influencia de la motivación deportiva en el consumo de alcohol, tabaco y cannabis en deportistas adolescentes escolares. De este modo, la primera hipótesis que planteada fue la existencia de relaciones significativas entre mayores motivaciones intrínsecas y menores consumos de alcohol, tabaco y cannabis en una línea más de conductas más autodeterminada.
Según desprenden los resultados, la hipótesis se cumplió; revelaron relaciones entre motivaciones predominantemente intrínsecas hacia la práctica, aprendizaje y perfeccionamiento con unas menores prevalencias de consumo de alcohol, tabaco y cannabis.

Moreno et al. (2008) encontraron que, aquellos deportistas con motivaciones más intrínsecas predecían positivamente los motivos de salud y ausencia de consumo de drogas. Así, los adolescentes con ausencia o escasa prevalencia de consumo se encontraban más intrínsecamente motivados hacia la práctica físico-deportiva que los orientados extrínsecamente. Diversos estudios asocian un menor consumo de tabaco y alcohol con una alta motivación intrínseca y mayor práctica físico-deportiva en edad extraescolar (Inglés et al., 2007; Llamas, 2009; Usán et al., 2017). En una misma línea, Leyton et al. (2012) manifiestan la relación de una ausencia de consumo de tabaco en relación con motivaciones intrínsecas unidas a hábitos de salud positivos, como un adecuado descanso y alimentación. De esta manera, la motivación se revela factor muy importante para la salud física y psicológica (Moreno et al. 2008; Portolés \& González, 2016; Rees $\&$ Sabia, 2010).

La segunda hipótesis del trabajo aludía a la existencia de relaciones significativas entre mayores motivaciones extrínsecas y falta de motivación con mayores consumos de alcohol, tabaco y cannabis en una línea de conductas menos autodeterminada. A tenor de los resultados, se demostró tal relación entre la motivación y el consumo de alcohol, tabaco y cannabis. Así, desde una línea menos autodeterminada diferentes trabajos aluden a una mayor medida de los motivos extrínsecos en la práctica físicodeportiva en coincidencia con el consumo de sustancias ligado a perfiles conductuales más desadaptativos (RuizRisueño, 2011; Ruiz-Juan \& Zamarripa, 2012; Vasters \& Pillon, 2011).

Los trabajos de Moreno et al. (2008) determinan que aquellos deportistas que poseen menos motivación autodeterminada en la práctica deportiva predicen significativamente hábitos de salud poco consistentes que conllevan el consumo de sustancias durante la adolescencia. De igual manera que los 
adolescentes de este estudio, los deportistas que consumen habitualmente alcohol y tabaco reflejan motivaciones predominantemente extrínsecas. Moreno y Cervelló (2009) muestran relaciones positivas entre motivaciones puramente extrínsecas y consumo de alcohol en comportamientos más desadaptativos. A su vez, el consumo de cannabis se ha relacionado peyorativamente con la motivación y adhesión hacia la práctica deportiva. De este modo, Villalbí et al. (2011) manifiestan la implicación y consecuencias del consumo de cannabis en el rendimiento deportivo de los futbolistas mermando su motivación intrínseca hacia la práctica deportiva.

Portodoello,la motivación delos adolescentes influye y desempeña una labor relevante sobre las conductas de consumo (Jiménez, Cervelló, García-Calvo, SantosRosa \& Iglesias, 2007; Rubak, Sandbaek, Lauritzen \& Christensen, 2005), por lo que resulta fundamental atender a ella para en la prevención e intervención del consumo en aras de una mayor adherencia y disfrute de la actividad físico-deportiva (Agulló-Calatayud, 2008; González-Alcaide, Valderrama-Zurián, y AleixandreBenavent, 2008; Bartík, 2012; Hodge, Hargreaves, Gerrard \& Lonsdale, 2013;Pérez-Milena, Olmedilla, Mesa, Jiménez, Martínez \& Pérez-Milena, 2010; Prieto, 2015; Roessler, 2009).

\section{Limitaciones del estudio}

Las limitaciones del estudio residen en su diseño transversal, pues toma los datos en un momento espacio temporal dado. A su vez, los clubes encuestados responden de una manera aleatoria a su inclusión en el estudio y no se toma una muestra uniforme de todos los barrios de la ciudad, por lo que se afecta a las posibles diferencias entre sus niveles deportivos y sociales. A la par, las prevalencias de motivación y consumo pueden variar de un año a otro, incluso dentro de un mismo año, en cuanto los adolescentes avanzan en la etapa de la adolescencia.

\section{Implicaciones prácticas}

Para finalizar, este trabajo puede tener unas implicaciones de carácter práctico que desemboquen en unas estrategias didácticas a utilizar sobre deportistas adolescentes. Lo anterior a través de entrenadores o monitores deportivos, que guíen hacia conductas autodeterminadas como pueden ser la promoción de motivaciones intrínsecas hacia la práctica del deporte impulsadas por el esfuerzo, interés y diversión en detrimento de las extrínsecas así como formación en consumo de sustancias. Un ejemplo de ello resultan las estrategias a utilizar para alcanzar un clima motivacional implicante a la tarea (García-Calvo, Santos-Rosa, Jiménez \& Cervelló, 2006).

A su vez, programas de intervención dirigidos por profesionales delámbito en la apuesta de la Administración y los propios clubes deportivos por el trabajo con sus adolescentes en las variables acaecidas puede ayudar a contribuir a la formación integral y educacional del deportista no reduciéndola sólo a la deportiva.

\section{Referencias}

Agulló-Calatayud, V., González-Alcaide, G., Valderrama-Zurián, J.C. \& Aleixandre-Benavent, R. (2008). Consumption of anabolic steroids in sport, physical activity and as a drug of abuse: An analysis of the scientific literature and areas of research. British Journal of Sports Medicine, 42(2), 103-109.

Alcalá, M., Azañas, S., Moreno, C., \& Gálvez, L. (2002). Consumo de alcohol, tabaco y otras drogas en adolescentes, estudio de dos cortes. Medicina de Familia, 3(2), 81-87.

Almagro, B. J., Sáenz, P., González-Cutre, D., \& Moreno, J.A. (2011). Clima motivacional percibido, necesidades psicológicas y motivación intrínseca como predictores del compromiso deportivo en adolescentes. Revista Internacional de Ciencias del Deporte, 25(7), 250-265. doi: 10.5232/ricyde2011.02501

Asociación Médica Mundial (2000). Declaración de Helsinki. Principios éticos para las investigaciones médicas en seres humanos. 52 Asamblea General de Edimburgo, Escocia. Octubre de 2000. Revista Gaceta Médica de México, 137(4): 387-390.

Ato, M., López, J., \& Benavente, A. (2013). Un sistema de clasificación de los diseños de investigación en psicología. Anales de psicología, 29(3), 1038-1059. 
Baquero, A., Pastor R., \& Llopis J. J. (2003). Interacciones y consecuencias del consumo combinado de alcohol y cocaína: Una actualización sobre el cocaetileno. Adicciones, 15(2), 159-164.

Bartik, P. (2012). Motor and sports activities as a good means against of drug use. Journal of Human Sport and Exercise, 7 (1 SPECIAL ISSUE), 147-153.

Becoña,E.(2007).Basespsicológicasdelaprevencióndel consumo de drogas. Papeles del Psicólogo, 28(1), 11-20.

Boyle, A. M., \& O’Sullivan, L. F. (2013). The influence of dating anxiety on normative experiences of dating, sexual interactions, and alcohol consumption among canadians middle adolescents. Journal of Youth Studies, 16 (2), 222-236. doi: 10.1080/13676261.2012.704987

Bravo, R., Echeburúa, E., \& Aizpiri, J. (2008). Diferencias de sexo en la dependencia del alcohol: dimensiones de personalidad, características psicopatológicas y trastornos de personalidad. Psicothema, 20, 218-223.

Calafat, A., Juan, M., Becoña J., Fernández, C., \& Gil, E. (2002). Salir de marcha y consumo de drogas. Madrid: Ministerio del Interior. Delegación del Gobierno para el Plan Nacional sobre Drogas.

Castillo, I., Balaguer, I., \& Duda, J. (2002). Las perspectivas de meta de los adolescentes en el contexto deportivo. Psicothema, 14(2), 280-287.

Cecchini, J. A., Gónzalez, C., Carmona A. M., \& Contreras O. (2004). Relaciones entre clima motivacional, la orientación de meta, la motivación intrínseca, la auto-confianza, la ansiedad y el estado de ánimo en jóvenes deportistas. Psicothema, 16(1), 104-109.

Cruz, P. J., Fernández, E., \& González, G. M. (2007). El deporte como método preventivo en el uso de las drogas: teoría y práctica. Revista Digital Wanceulen, 3. Recuperado de: http://www.wanceulen.com/ revista/PDF/n3/deporte_preventivo_drogas.pdf

De Vries, H., Dijk, F.,Wetzels, J., Mudde, A., Kremers, S., \&Ariza C. (2006). The European Smokingprevention
Framework Approach (ESFA), effects after 24 and 30 months. Health Education Research, 21(1), 116-132.

Deci, E. L., \& Ryan, R. M. (2000). The "what" and "why" of goal pursuits: Human needs and the selfdetermination of behavior. Psychological Inquiry, 11, 227-268.

Deci, E. L., \& Ryan, R. M. (2002). Self-determination research: Reflections and future directions. In E.L. Deci y R.M. Ryan (Eds.), Handbook of selfdetermination research. (pp. 431-441). University of Rochester Press.

Dunn, M., \& Thomas, J. O. (2012). A risk profile of elite australian athletes who use illicit drugs. Addictive Behaviors, 37(1), 144-147.

Encuesta Estatal sobre Uso de Drogas en Enseñanzas Secundarias (ESTUDES). (2013). Madrid: Ministerio del Interior. Plan Nacional sobre Drogas.

Espada, J., Méndez, X., Griffin, K. \& Botvin, G. (2003). Adolescencia: Consumo de alcohol y otras drogas. Papeles del psicólogo, 84, 9-17.

Fox, K. R., \& Corbin, C. B. (1989). The physical selfperception profile: Development and preliminary validation. Journal of Sport and Exercise Psychology, 11, 408-430.

García, P., Carrillo, A., Fernández, A., \& Sánchez, J. M. (2006). Factores de riesgo en la experimentación y el consumo de tabaco en estudiantes de 12 a 14 años. Actitudes ante el tabaco en los grupos de presión. Revista de Atención Primaria, 37, 392-399.

García, P., Cuevas, M. D., Hurtado, S., Sánchez, J., \& Martínez, R. (2005). Comparación de las actitudes, percepción, entorno y experimentación con el tabaco en cuatro poblaciones de $1^{\circ}$ de ESO de la Región de Murcia. I Congreso Internacional de Enfermería Comunitaria, Madrid.

García-Calvo, T. (2006). Motivación y comportamientos adaptativos en jóvenes futbolistas. Tesis Doctoral. Universidad de Extremadura, España.

García-Calvo, T., Santos-Rosa, F.J., Jiménez, R., \& Cervelló, E.M. (2006). El clima motivacional en las clases de Educación Física: una aproximación 
práctica desde la Teoría de Metas de Logro. Apuntes: Educación física y deportes, 81, 21-28.

Gilman, S. E., Rende, R., Boergers, J., Abrams, D. B., Buka, S. L., Clark, M. A., \& Niaura, R. S. (2009). Parental smoking and adolescent smoking initiation: an intergenerational perspective on tobacco control. Pediatrics, 123, 274-281.

Gómez-Fraguela, J. A., Fernández, N., Romero, E., \& Luengo, A. (2008). El botellón y el consumo de alcohol y otras drogas en la juventud. Trastornos Adictivos, 20(2), 211-217.

Goncy, E. A., \& Mrug, S. (2013). Where and when adolescents use tobacco, alcohol, and marijuana: Comparisons by age, gender, and race. Journal of Studies on Alcohol and Drugs, 74(2), 288-300.

González-Cutre, D., Sicilia, A., \& Moreno, J. A. (2006). Predicción de las razones de responsabilidad para ser disciplinado en el deporte a través de las orientaciones de meta y los climas motivacionales. En M. A. González, J. A. Sánchez y A. Areces (Eds.), IV Congreso de la Asociación Española de Ciencias del Deporte (pp. 736-739). A Coruña: Xunta de Galicia.

Goñi, A., Ruiz de Azúa, S., \& Rodríguez, A. (2006). Cuestionariode AutoconceptoFísico. Manual.Madrid:EOS.

Hellín, M. G. (2007). Motivación, autoconcepto físico, disciplina y orientación disposicional en estudiantes de educación física. Tesis Doctoral. Universidad de Murcia.

Hock, H., \& Weil, D.N. (2012). On the dynamics of the age structure, dependency, and consumption. Journal of Population Economics, 25(3), 1019-1043.

Hodge, K., Hargreaves, E.A., Gerrard, D. \& Lonsdale, C. (2013). Psychological mechanisms underlying doping attitudes in sport: Motivation and moral disengagement. Journal of Sport and Exercise Psychology, 35(4), 419-432.

Holgado, F. P., Soriano, J. A., \& Navas L. (2009). Cuestionario de autoconcepto físico (CAF): Análisis factorial confirmatorio y predictivo sobre el rendimiento académico global y específico del área de educación física. Acción psicológica, 6(2), 93-102.
Inglés, C.J., Delgado, B., Bautista, R., Torregrosa, M.S., Espada, J.P., García-Fernández, J.M., Hidalgo, M.D. \& García-López, L.J. (2007). Factores psicosociales relacionados con el consumo de alcohol y tabaco en adolescentes españoles. International Journal of Clinical and Health Psychology, 7, 403-420.

Iso-Ahola, S. E., \& St.Clair, B. (2008). Toward a theory of exercise motivation. Quest, 52, 131-147.

Jiménez, R., Cervelló, E., García-Calvo, T., SantosRosa, F.J., \& Iglesias, D. (2007). Estudio de las relaciones entre motivación, práctica deportiva extraescolar y hábitos alimenticios y de descanso en estudiantes de Educación Física. International Journal of Clinical and Health Psychology, 7(2), 385-401.

Llamas, L.S. (2009). Motivación autodeterminada y clima motivacional en educación física. Tesis Doctoral. Universidad de Elche.

Leyton, M., Jiménez, R., Domínguez, F.J. \& Corzo., H. (2012). Análisis correlacional entre la teoría de la autodeterminación y variables de estilos de vida saludables: descanso, tabaco y alimentación. IV Congreso internacional de ciencias del deporte y la Educación Física. (VIII Seminario nacional de nutrición, medicina y rendimiento deportivo). Pontevedra.

Macarro, J., Romero, C., \& Torres, J. (2008). Motivos de abandono de la práctica de actividad físico-deportiva en los estudiantes de Bachillerato de la provincia de Granada. Revista de Educación, 353, 495-519.

Mañas, M. A., Salvador, C., Boada, J., \& Agulló, A. (2007). La satisfacción y el bienestar psicológico como antecedentes del compromiso organizacional. Psicothema, 19, 395-400.

Moreno, J.A., González-Cutre, D. \& Cervelló, E.M. (2008). Motivación y salud en la práctica físicodeportiva: diferencias según el consumo de alcohol y tabaco. International Journal of Clinical and Health Psychology, 8, 483-494.

Moreno, J. A., \& Martínez, A. (2006). Importancia de la Teoría de la Autodeterminación en la práctica físicodeportiva: Fundamentos e implicaciones prácticas. Cuadernos de Psicología del Deporte, 6(2), 39-54. 
Moreno, J.A., Moreno, R. \& Cervelló, E. (2009). Relación del autoconcepto físico con las conductas de consumo de alcohol y tabaco en adolescentes. Adicciones, 21, 147-154.

Nistal, P., Prieto, J.A., Del Valle, M. \& González, V. (2003) Relación de la actividad física con el consumo de tabaco en adolescentes. Archivos de medicina del deporte, 20(97), 397-403.

Núñez, J.L., Martín-Albo, J., Navarro, J.G. \& González, V.M. (2006). Preliminary validation of a Spanish version of the Sport Motivation Scale. Perceptual and Motor Skills, 102, 919-930.

Oliva, A., Parra A., \& Sánchez-Queija, I. (2006). Consumo de sustancias durante la adolescencia: Trayectorias evolutivas y consecuencias para el ajuste psicológico. International Journal of Clinical and Health Psychology, 8(1), 153-169.

Pastor, Y., Balaguer, I. \& García-Merita, M. (2006). Relaciones entre el autoconcepto y el estilo de vida saludable en la adolescencia media: un modelo exploratorio. Psicothema, 18(1), 18-24.

Pelletier, L. G., Fortier, M. S., Vallerand, R. J., Tuson, K. M., Brière, N. M., \& Blais, M. R. (1995). Toward a new measure of intrinsic motivation, extrinsic motivation, and amotivation in sports: The Sport Motivation Scale (SMS). Journal of Sport \& Exercise Psychology, 17, 35-53.

Pérez-Milena, A., Olmedilla, M.R., Mesa, I., Jiménez, I., Martínez, M.L., \& Pérez-Milena, R. (2010). Motivaciones para el consumo de alcohol entre adolescentes de un instituto urbano. Atencion Primaria, 42(12), 604-611.

Plan Nacional Sobre Drogas (PNSD). (2016). Alcohol, tabaco $y$ otras drogas ilegales en España. Madrid: Ministerio del Interior.

Portolés, A. \& González, J. (2016). Actividad física y niveles de burnout en alumnos de la E.S.O. Retos. Nuevas tendencias en Educación Física, Deporte y Recreación, 29(1), 95-99.

Prieto, J.M. (2015). Variables deportivas y personales en la ocurrencia de lesiones deportivas. Diferencias entre deportes individuales y colectivos. Retos. Nuevas tendencias en Educación Física, Deporte y Recreación, 28(2), 21-25.

Rees, D.I. \& Sabia, J.J. (2010). Sports participation and academic performance: Evidence from the national longitudinal study of adolescent health. Economics of Education Review, 29 (5), 751-759.

Rodríguez J., De Abajo, S., \& Márquez, S. (2004). Relación entre actividad física y consumo de alcohol, tabaco y otras sustancias perjudiciales para la salud en alumnos de ESO del municipio de Avilés. European Journal of Human Movement, 12, 46-69.

Roessler, K. (2009). "Sport makes me high" The role of physical activity in treatment for drug abuse. Zeitschrift Fur Sportpsychologie, 16 (4), 151-155.

Rubak, S., Sandbaek, A., Lauritzen, T. \& Christensen, B. (2005). Motivational interviewing: A systematic review and meta-analysis. British Journal of General Practice, 55, 305-312.

Ruiz-Juan, J. M., Isorna, M., Ruiz-Risueño, J. R., \& Vaquero, R. (2014). Consumo e ingesta de alcohol en españoles mayores de 16 años y su relación con la actividad físico-deportiva, la familia y el consumo de tabaco. Revista Iberoamericana de Psicología del Ejercicio y el Deporte, 9(2), 339-372.

Ruiz-Risueño, J., Ruiz-Juan, F., \& Zamarripa, J.I. (2012). Alcohol y tabaco en adolescentes españoles y mexicanos y su relación con la actividad físico-deportiva y la familia. Panam Salud Publica, 31(3), 211-220.

Salomó A., Gras M. E., \& Font-Mayolas S. (2010). Patrones de consumo de alcohol en la adolescencia. Psicothema, 22(2), 189-95.

Scanlan, T. K., Russell, D. G., Beals, K. P., \& Scanlan, L. A. (2003). Project on Elite Athlete Commitment (PEAK): Introduction and Methodology. Journal of Sport and Exercise Psychology, 25, 360-376.

Shavelson, R. J., Hubner, J. J., \& Stanton, J. C. (1976). Self-concept: Validation of construct interpretations. Review of Elementary School Children, 46, 407-441. 
Torregrosa, M., Sousa, C., Viladrich., C., Villamartín., F., \& Cruz., J. (2008). El clima motivacional y el estilo de comunicación del entrenador como predictores del compromiso en futbolistas jóvenes. Psicothema, 20(2), 254-259.

Usán, P., Salavera, C., Murillo, V., \& Álvarez, J. (2017). Creencias y percepciones de éxito en futbolistas adolescentes. Retos. Nuevas Tendencias en Educación Física, Deporte y Recreación, 31(1), 207-211.

Vasters, G. P., \& Pillon, S. C. (2011). Drugs use by adolescents and their perceptions about specialized treatment adherence and dropout. Revista Latino Americana De Enfermagem, 19(2), 317-324.

Villalbí, J., Suelves, J., Saltó., E. \& Cabezas, C. (2011). Valoración de las encuestas a adolescentes sobre consumo de tabaco, alcohol y cannabis en España, Adicciones, 23(1), 11-16.

Warren, C. W., Jones, N. R., Peruga, A., Chauvin, J., Baptiste, J. P., \& Costa de Silva,V. (2008). Global Routh tobacco surveillance, 2000-2007. MMWR Surveill Summ, 57(1), 1-28.

Recibido: 16 de febrero de 2017 Aceptado: 9 de mayo de 2017 
